\title{
Broadband tunable nonlinear optical response in plasmonic metamaterials -INVITED
}

\author{
Tiziana Cesca ${ }^{1,{ }^{*}}$, Domenico Genchi ${ }^{1}$, Raul Rangel-Rojo ${ }^{2}$, Jorge A. Reyes-Esqueda ${ }^{3}$ and Giovanni Mattei ${ }^{1}$ \\ ${ }^{1}$ Physics and Astronomy Department, University of Padova, via Marzolo 8, I-35131 Padova, Italy \\ ${ }^{2}$ División de Física Aplicada, Centro de Investigación Científica y de Educación Superior de Ensenada, Ensenada, México \\ ${ }^{3}$ Instituto de Física, Universidad National Autónoma de México (UNAM), México City, México
}

\begin{abstract}
Nanostructured materials with tunable nonlinear optical response are of great interest for different applications in nanophotonics. In this work we report the results of a comprehensive study on the nonlinear optical properties of two kinds of plasmonic metamaterials, i.e., honeycomb nanoprism arrays and multilayer hyperbolic metamaterials, which proved to have a very rich spectrum of parameters (as metamaterials morphology and composition, wavelength and polarization of an input beam) to exploit for controlling their nonlinear response over a broad spectral range.
\end{abstract}

\section{Introduction}

In recent years, nanomaterials with properly engineered nonlinear optical properties have been the object of an intense scientific research for their application in different fields in nanophotonics. Within this framework, plasmonic metamaterials have attracted considerable attention due to their strong and ultra-fast nonlinear optical (NLO) response [1] and the possibility of tailoring their nonlinear optical properties by manipulating the metamaterials nanostructure [2, 3]. Besides this, an important requirement for NLO applications is the capability to tune the material nonlinearity in a reversible way, by changing some external control parameters.

In this work, we present the results of a comprehensive study on the nonlinear optical properties of two classes of plasmonic metamaterials, namely honeycomb, $\mathrm{Au}$ and Ag, nanoprism arrays (HNPA) and multilayer hyperbolic metamaterials (MHM). The dependence of the NLO properties on wavelength, irradiance, and polarization of the input beam, as well as on the metamaterials morphology and composition, is investigated by using the z-scan technique [4] in the picosecond and femtosecond regimes. The temporal dynamics of the nonlinear optical response is also investigated employing the time-resolved excite-probe technique [5]. Finally, the polarizationdependent second harmonic generation (SHG) properties of the HNPA are characterized [6]. The experimental findings are compared and interpreted on the basis of the results of finite elements methods (FEM) simulations of the plasmonic properties (near-field and far-field) of the investigated nanosystems.

\section{Experimental}

Honeycomb $\mathrm{Au}$ and Ag nanoprism arrays (NPA) are fabricated on silica substrates by nanosphere lithography (NSL) and thermal evaporation. Thermal annealing treatments in air, at different temperatures, are done to modify the shape of the nanostructures (from triangular nanoprisms to hemispherical nanoparticles) without altering the array periodicity. Multilayer hyperbolic metamaterials (MHM) are produced by magnetron sputtering depositions of alternate metallic and dielectric layers. MHMs with different metallic filling fraction are obtained by changing the relative thickness of the dielectric and metallic layers.
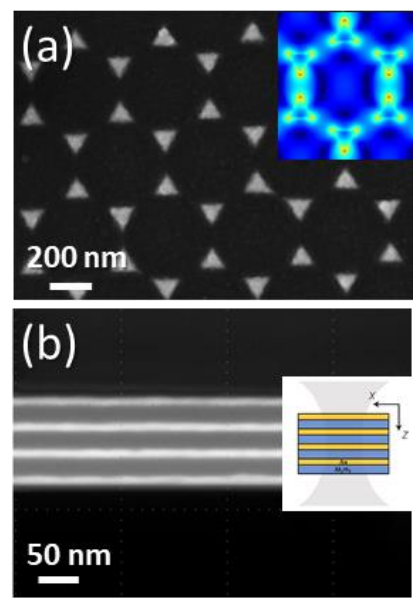

Fig. 1. (a) SEM image in plane-view of an Ag honeycomb nanoprism array (HNPA). Inset: local-field enhancement computed at the dipolar surface plasmon resonance of the HNPA. (b) SEM image in cross-section of an $\mathrm{Au} / \mathrm{Al}_{2} \mathrm{O}_{3}$ multilayer hyperbolic metamaterial (MHM). Inset: sketch of the sample.

\footnotetext{
* Corresponding author: tiziana.cesca@unipd.it
} 
Fig. 1 shows an example of both kinds of synthesized metamaterials: (a) Ag HNPA and (b) $\mathrm{Au} / \mathrm{Al}_{2} \mathrm{O}_{3} \mathrm{MHM}$.

Finite elements method (FEM) electrodynamic simulations of the near-field and far-field optical properties of the metamaterials are carried out with the commercial software COMSOL Multiphysics.

The spectral characterization of the nonlinear optical properties of the metamaterials was performed with the $\mathrm{z}-$ scan technique [4] using a mode-locked ps Nd:YAG laser (pulse duration $<20 \mathrm{ps}, 10 \mathrm{~Hz}$ repetition rate), coupled to an optical parametric amplifier. A fs mode-locked Ti:sapphire laser (125 fs, $100 \mathrm{MHz})$ was used to investigate the ultra-fast dynamics of the nonlinear response of the HNPA, and to characterize their second harmonic generation (SHG) properties.

\section{Results and discussion}

Annealing treatments in air at different temperatures are used to change the morphology of the nanostructures in the plasmonic HNPA, varying their shape from triangular nanoprisms to hemispherical nanoparticles. This allows us to control the linear optical properties of the samples, tuning the spectral position and amplitude of their surface plasmon resonance peak and, in this way, to modulate their nonlinear optical parameters (nonlinear refractive index $\mathrm{n}_{2}$, and nonlinear absorption coefficient $\beta$ ). As an example, Fig. 2 shows the evolution, as a function of the annealing temperature, of $\mathrm{n}_{2}$ for an Au HNPA. The experimental findings are well described by FEM simulations of the local-field distribution in the nanoarrays [7].

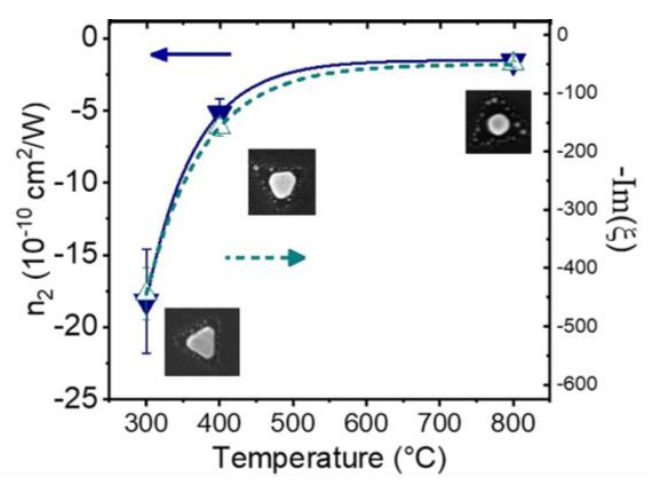

Fig. 2. Nonlinear refractive index $\mathrm{n}_{2}$ (blue triangles, left-hand scale) of Au HNPA as a function of the annealing temperature. Open symbols (right-hand scale): imaginary component of the integrated local-field distribution in the nanoarray.

The angle of incidence and the polarization orientation of an input beam can be used instead to get a reversible and continuous modulation of the NLO response of the synthesized metamaterials. As an example, in Fig. 3(a) we report the spectral trend of the nonlinear absorption coefficient $(\beta)$ of an $\mathrm{Au} / \mathrm{Al}_{2} \mathrm{O}_{3}$ MHM (Au filling fraction $16 \%$ ), at different incidence angles for TE polarization. Fig. 3(b) shows the trend of $\beta$, as a function of the linear polarization orientation of the input beam, of an $\mathrm{Au}$ HNPA: the trend has the $60^{\circ}$-symmetry of the honeycomb lattice and it is correlated to the polarization-dependent local electric-field distribution in the nanoarray, as demonstrated by FEM simulations [8].
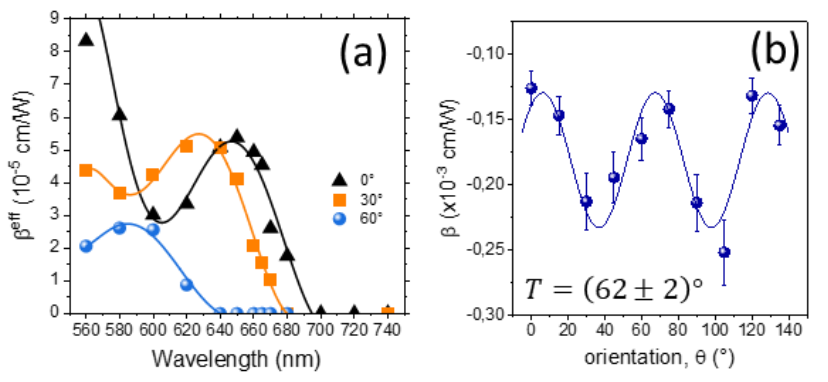

Fig. 3. (a) Spectral trend of the nonlinear absorption coefficient ( $\beta$ ) of an $\mathrm{Au} / \mathrm{Al}_{2} \mathrm{O}_{3} \mathrm{MHM}$ measured at different incidence angles for TE polarization. (b) $\beta$ as a function of the input linear polarization orientation of an Au HNPA.

\section{Conclusions}

The nonlinear optical properties of two classes of plasmonic metamaterials, namely honeycomb nanoprism arrays and multilayer hyperbolic metamaterials, have been investigated as a function of different parameters, as morphology and composition of the nanosystems or polarization and spectral range of the input beam. The results demonstrate that these metamaterials offer several degrees of freedom to tailor their nonlinear optical response, and a reversible and continuous modulation of their nonlinear optical properties can be obtained.

\section{References}

1. M. Kauranen, A. V. Zayats, Nat. Photon. 6, 737 (2012)

2. T. Cesca, N. Michieli, B. Kalinic, I. G. Balasa, R. Rangel-Rojo, J.A. Reyes Esqueda, G. Mattei, Mat. Sci Semicon. Proc. 92, 2 (2019)

3. T. Cesca, N. Michieli, B. Kalinic, A. SanchezEspinoza, M. Rattin, V. Russo, V. Mattarello, C. Scian, P. Mazzoldi, G. Mattei, Nanoscale 7, 12411 (2015)

4. M. Sheik-Bahae, A.A. Said, T.H. Wei, D.J. Hagan, E.W. Van Stryland, IEEE Journal of Quantum Electronics 26, 760 (1990)

5. H. Sanchez-Esquivel, K.Y. Raygoza-Sanchez, R. Rangel-Rojo, B. Kalinic, N. Michieli, T. Cesca, G. Mattei, Nanoscale 10, 5182 (2018)

6. K.Y. Raygoza-Sanchez, I. Rocha-Mendoza, P. Segovia, A.V. Krasavin, G. Marino, T. Cesca, N. Michieli, G. Mattei, A.V. Zayats, R. Rangel-Rojo, Sci. Rep. 9, 11514 (2019)

7. T. Cesca, M. Manca, N. Michieli, G. Mattei, Appl. Surf. Sci. 491, 67 (2019)

8. T. Cesca, E.V. Garcia Ramirez, H. Sanchez-Esquivel, N. Michieli, B. Kalinic J.M. Gomez Cervantes, R. Rangel-Rojo, J.A. Reyes-Esqueda, G. Mattei, RSA Adv. 7, 17741 (2017) 